\title{
Antigua and Barbuda: Statistical Appendix
}

This Statistical Appendix for Antigua and Barbuda was prepared by a staff team of the International Monetary Fund as background documentation for the periodic consultation with the member country. It is based on the information available at the time it was completed on December 8, 2006. The views expressed in this document are those of the staff team and do not necessarily reflect the views of the government of Antigua and Barbuda or the Executive Board of the IMF.

The policy of publication of staff reports and other documents by the IMF allows for the deletion of market-sensitive information.

To assist the IMF in evaluating the publication policy, reader comments are invited and may be sent by e-mail to publicationpolicy@imf.org.

Copies of this report are available to the public from

International Monetary Fund $\bullet$ Publication Services

700 19th Street, N.W. • Washington, D.C. 20431

Telephone: (202) 6237430 • Telefax: (202) 6237201

E-mail: publications@imf.org • Internet: http://www.imf.org

Price: $\$ 18.00$ a copy

\section{International Monetary Fund Washington, D.C.}





\section{INTERNATIONAL MONETARY FUND}

\section{ANTIGUA AND BARBUDA}

\section{Statistical Appendix}

Prepared by a staff team consisting of Ulric Erickson von Allmen, Rupa Duttagupta, Dmitriy Kovtun, and Koffie Nassar

Approved by Western Hemisphere Department

December 8, 2006

Contents

Page

Basic Data.

Tables

1. GDP by Sector of Origin at Current Prices, 2001-05 3

2. GDP by Sector of Origin at Constant 1990 Prices, 2001-05 ..........................................

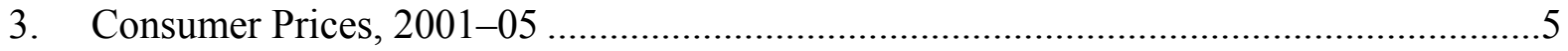

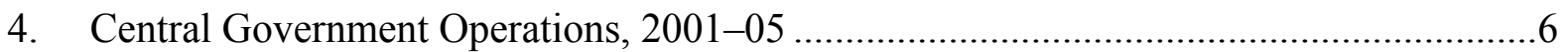

5. Central Government Revenue and Grants, 2001-05 ..................................................

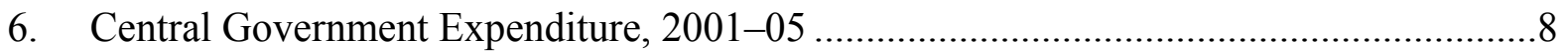

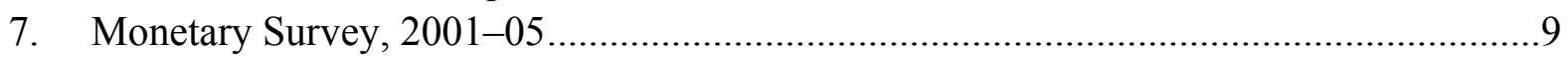

8. Summary Operations of the Eastern Caribbean Central Bank, 2001-05..........................10

9. Consolidated Accounts of the Commercial Banks, 2001-05 ..........................................11

10. Distribution of Commercial Bank Loans and Advances, 2001-05 ................................12

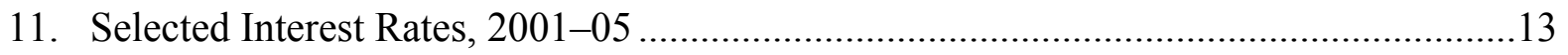

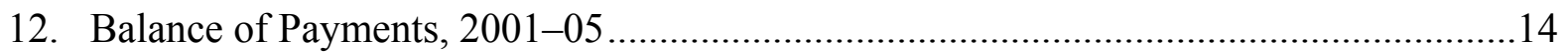

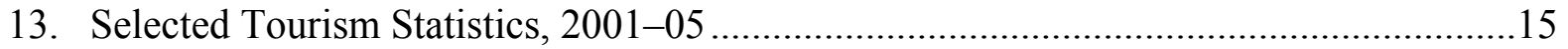

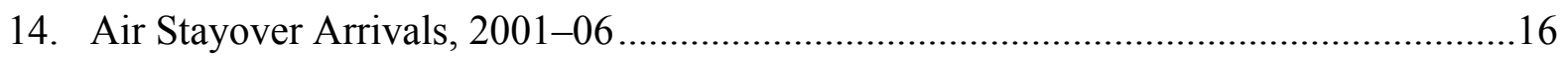

15. Air Stayover Arrivals by Country of Origin, 2001-05 ...............................................17

16. Visitor Arrivals as a Share of the Caribbean Area, 2001-05 ..........................................18

17. Public and Publicly Guaranteed External Debt, 2001-05 .............................................19

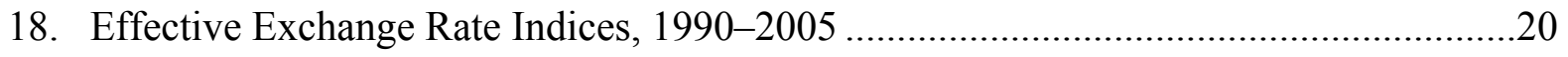


Antigua and Barbuda: Basic Data

Area (sq. km)

Arable land (percent of land area)

Population (2001)

Total (thousand)

Annual rate of growth, 1991-2001

(percent a year)

Density (per sq. km.)

GDP per capita (US\$), 2004

Population characteristics (1999)

Life expectancy at birth (years)

Crude birth rate (per thousand)

Crude death rate (per thousand)

I. Social and Demographic Indicators

27.3 Population per physician

Population per hospital bed

Education (2001; in percent)
Adult literacy rate

$1.0 \quad$ Energy (2005; in millions of US\$)

10,317 Imports of petroleum products

$75 \quad$ GDP (2005)

$19 \quad$ (in millions of EC\$) 2,362

$8 \quad$ (in millions of US\$)

II. Selected Economic and Financial Indicators, 2002-07

\begin{tabular}{|c|c|c|c|c|c|}
\hline & 2001 & 2002 & 2003 & 2004 & $\begin{array}{r}\text { Est. } \\
2005 \\
\end{array}$ \\
\hline & \multicolumn{5}{|c|}{ (Annual percentage changes, unless otherwise specified) } \\
\hline \multicolumn{6}{|l|}{ National income and prices } \\
\hline GDP at constant factor cost & 1.5 & 2.0 & 4.3 & 5.2 & 5.3 \\
\hline Nominal GDP at market prices & 4.7 & 1.0 & 5.1 & 8.5 & 6.9 \\
\hline Consumer prices (end of period) 1/ & $\ldots$ & 2.6 & 1.8 & 2.8 & 2.5 \\
\hline Consumer prices (period average) $1 /$ & $\ldots$ & 2.4 & 2.0 & 2.0 & 2.1 \\
\hline \multicolumn{6}{|l|}{ External sector } \\
\hline Exports, f.o.b. & -12.6 & -24.7 & 32.9 & 27.7 & 25.8 \\
\hline Imports, f.o.b. & 5.2 & -4.6 & 16.3 & 6.3 & 10.9 \\
\hline Travel receipts (gross) & -6.4 & 0.6 & 9.5 & 12.6 & -0.3 \\
\hline Nominal effective exchange rate (period ave., depreciation -) & 2.5 & -0.3 & -3.2 & -2.7 & -0.3 \\
\hline \multirow[t]{2}{*}{ Real effective exchange rate (period ave., depreciation -) } & 3.3 & 1.3 & -2.2 & -2.2 & -0.3 \\
\hline & \multicolumn{5}{|c|}{ (Contribution to broad money growth) } \\
\hline \multicolumn{6}{|l|}{ Money and credit } \\
\hline Net foreign assets & 12.6 & 0.6 & 21.9 & 3.3 & 30.0 \\
\hline Net domestic assets & -7.8 & 5.7 & -0.8 & 5.9 & -23.1 \\
\hline Net credit to the public sector & -1.8 & 0.3 & 3.0 & -1.1 & -1.2 \\
\hline Credit to the private sector & 2.9 & 7.5 & 2.2 & 0.9 & 7.7 \\
\hline Broad money & 4.8 & 6.3 & 21.1 & 9.2 & 6.9 \\
\hline Average deposit rate (in percent per annum) 2/ & 4.5 & 4.4 & 4.9 & 4.1 & 3.9 \\
\hline \multirow[t]{2}{*}{ Average lending rate (in percent per annum) $2 /$} & 11.6 & 11.4 & 12.8 & 12.0 & 11.4 \\
\hline & \multicolumn{5}{|c|}{ (In percent of GDP) } \\
\hline \multicolumn{6}{|l|}{ Central government } \\
\hline Primary balance & -6.8 & -6.9 & -4.2 & -1.2 & -2.3 \\
\hline Overall balance & -12.4 & -12.2 & -9.5 & -6.1 & -7.1 \\
\hline Total revenue and grants & 19.2 & 21.7 & 21.1 & 21.6 & 21.8 \\
\hline Total expenditure and net lending & 31.6 & 33.9 & 30.7 & 27.7 & 28.9 \\
\hline Identified financing & 5.5 & 6.8 & 11.9 & 6.4 & 8.5 \\
\hline External & 3.9 & 3.2 & 7.3 & 0.1 & -20.9 \\
\hline Domestic & 1.5 & 3.5 & 4.5 & 4.5 & 8.0 \\
\hline Statistical discrepancy & 6.9 & 5.5 & -2.3 & -0.3 & -1.3 \\
\hline \multicolumn{6}{|l|}{ External sector } \\
\hline Current account balance & -8.0 & -10.9 & -13.4 & -9.5 & -14.5 \\
\hline Trade balance & -38.4 & -37.5 & -40.8 & -38.8 & -39.3 \\
\hline Service balance & 32.6 & 31.7 & 30.7 & 33.0 & 29.7 \\
\hline \multicolumn{6}{|l|}{ Of which } \\
\hline Gross tourism receipts & 38.3 & 38.1 & 39.8 & 41.3 & 38.5 \\
\hline Overall balance & -0.9 & -1.6 & -1.6 & -2.1 & -15.8 \\
\hline External government debt (end of year) & 66.6 & 73.2 & 78.0 & 70.8 & 40.1 \\
\hline \multicolumn{6}{|l|}{ Of which } \\
\hline Arrears & 14.5 & 19.0 & 23.2 & 22.5 & 16.4 \\
\hline \multirow{3}{*}{$\begin{array}{l}\text { Scheduled external debt service } 3 / \\
\text { (In percent of exports of goods and services) }\end{array}$} & & & & & \\
\hline & 8.4 & 8.5 & 8.9 & 9.7 & 30.8 \\
\hline & \multicolumn{5}{|c|}{ (In millions of U.S. dollars, end of period) } \\
\hline \multicolumn{6}{|l|}{ Gross international reserves of the ECCB } \\
\hline (In millions of U.S. dollars) & 446.0 & 504.8 & 539.9 & 632.4 & 600.8 \\
\hline (In percent of ECCU broad money) & 19.1 & 20.2 & 19.8 & 20.5 & 17.9 \\
\hline Nominal GDP at market prices (in millions of EC\$) & 1,918 & 1,938 & 2,036 & 2,210 & 2,362 \\
\hline \multicolumn{6}{|l|}{ Total government debt } \\
\hline (In millions of EC\$) & 2,482 & 2,716 & 2,989 & 3,035 & 2,543 \\
\hline (In percent of GDP) & 129 & 140 & 147 & 137 & 108 \\
\hline
\end{tabular}

Sources: Antigua and Barbuda authorities; Eastern Caribbean Central Bank; and Fund staff estimates and projections.

1/ Based on preliminary estimates of an unpublished, new consumer price index, beginning January 2001, compiled by the Statistics Division, Ministry of Finance.

$2 /$ There is a break in the interest rate series in 2003 , owing to changes in reporting requirements for banks.

3/ Data for 2005 includes debt relief from the Italian government. 
Table 1. Antigua and Barbuda: GDP by Sector of Origin at Current Prices, 2001-05

\begin{tabular}{|c|c|c|c|c|c|}
\hline & 2001 & 2002 & 2003 & 2004 & $\begin{array}{l}\text { Prel. } \\
2005 \\
\end{array}$ \\
\hline & \multicolumn{5}{|c|}{ (In millions of Eastern Caribbean dollars) } \\
\hline GDP at current market prices & $1,918.2$ & $1,938.2$ & $2,036.2$ & $2,209.8$ & $2,362.2$ \\
\hline (In millions of U.S. dollars) & 710.5 & 717.9 & 754.1 & 818.5 & 874.9 \\
\hline Net indirect taxes & 298.0 & 286.3 & 307.7 & 369.3 & 391.8 \\
\hline GDP at factor cost & $1,620.2$ & $1,651.9$ & $1,728.4$ & $1,840.6$ & $1,970.5$ \\
\hline Agriculture & 61.6 & 62.6 & 65.2 & 67.9 & 71.4 \\
\hline Crops & 16.6 & 17.1 & 17.7 & 18.6 & 19.5 \\
\hline Livestock & 13.0 & 13.5 & 14.0 & 14.4 & 14.9 \\
\hline Forestry & 0.9 & 0.9 & 0.9 & 0.9 & 0.9 \\
\hline Fisheries & 31.0 & 31.2 & 32.6 & 34.1 & 36.0 \\
\hline Mining and quarrying & 27.3 & 28.3 & 29.8 & 4.5 & 5.2 \\
\hline Manufacturing & 36.7 & 38.1 & 40.1 & 39.4 & 39.8 \\
\hline Electricity and water & 62.8 & 51.4 & 50.6 & 52.9 & 57.7 \\
\hline Construction & 216.5 & 228.6 & 243.9 & 254.9 & 319.9 \\
\hline Transportation & 189.3 & 193.1 & 215.0 & 253.0 & 252.8 \\
\hline Road transport & 83.6 & 77.7 & 87.9 & 101.9 & 103.9 \\
\hline Sea transport & 23.6 & 22.5 & 32.0 & 39.1 & 36.1 \\
\hline Air transport & 82.1 & 93.0 & 95.1 & 111.9 & 112.8 \\
\hline Communication & 137.4 & 137.9 & 140.1 & 145.5 & 157.2 \\
\hline Trade & 169.0 & 167.4 & 177.4 & 185.8 & 195.7 \\
\hline Hotels and restaurants & 164.6 & 157.3 & 173.4 & 185.2 & 194.1 \\
\hline Banks and insurance & 143.4 & 161.4 & 158.8 & 174.1 & 176.3 \\
\hline Housing & 115.3 & 120.6 & 126.7 & 133.7 & 141.2 \\
\hline Government services & 281.8 & 297.8 & 306.5 & 320.5 & 330.1 \\
\hline Other services & 119.3 & 124.8 & 126.7 & 133.7 & 138.9 \\
\hline \multirow[t]{2}{*}{ Less: imputed banking services } & 104.5 & 117.2 & 125.8 & 132.3 & 137.6 \\
\hline & \multicolumn{5}{|c|}{ (In percent of GDP at factor cost) } \\
\hline GDP at factor cost & 100.0 & 100.0 & 100.0 & 100.0 & 100.0 \\
\hline Agriculture & 3.8 & 3.8 & 3.8 & 3.7 & 3.6 \\
\hline Crops & 1.0 & 1.0 & 1.0 & 1.0 & 1.0 \\
\hline Livestock & 0.8 & 0.8 & 0.8 & 0.8 & 0.8 \\
\hline Forestry & 0.1 & 0.1 & 0.1 & 0.0 & 0.0 \\
\hline Fisheries & 1.9 & 1.9 & 1.9 & 1.9 & 1.8 \\
\hline Mining and quarrying & 1.7 & 1.7 & 1.7 & 0.2 & 0.3 \\
\hline Manufacturing & 2.3 & 2.3 & 2.3 & 2.1 & 2.0 \\
\hline Electricity and water & 3.9 & 3.1 & 2.9 & 2.9 & 2.9 \\
\hline Construction & 13.4 & 13.8 & 14.1 & 13.9 & 16.2 \\
\hline Transportation & 11.7 & 11.7 & 12.4 & 13.7 & 12.8 \\
\hline Road transport & 5.2 & 4.7 & 5.1 & 5.5 & 5.3 \\
\hline Sea transport & 1.5 & 1.4 & 1.9 & 2.1 & 1.8 \\
\hline Air transport & 5.1 & 5.6 & 5.5 & 6.1 & 5.7 \\
\hline Communication & 8.5 & 8.3 & 8.1 & 7.9 & 8.0 \\
\hline Trade & 10.4 & 10.1 & 10.3 & 10.1 & 9.9 \\
\hline Hotels and restaurants & 10.2 & 9.5 & 10.0 & 10.1 & 9.9 \\
\hline Banks and insurance & 8.8 & 9.8 & 9.2 & 9.5 & 8.9 \\
\hline Housing & 7.1 & 7.3 & 7.3 & 7.3 & 7.2 \\
\hline Government services & 17.4 & 18.0 & 17.7 & 17.4 & 16.8 \\
\hline Other services & 7.4 & 7.6 & 7.3 & 7.3 & 7.0 \\
\hline \multirow[t]{2}{*}{ Less: imputed banking services } & 6.4 & 7.1 & 7.3 & 7.2 & 7.0 \\
\hline & \multicolumn{5}{|c|}{ (Annual percentage change) } \\
\hline GDP at current market prices & 4.7 & 1.0 & 5.1 & 8.5 & 6.9 \\
\hline GDP at current factor cost & 4.1 & 2.0 & 4.6 & 6.5 & 7.1 \\
\hline
\end{tabular}

Sources: Antigua and Barbuda authorities; Eastern Caribbean Central Bank; and Fund staff estimates. 
Table 2. Antigua and Barbuda: GDP by Sector of Origin at Constant 1990 Prices, 2001-05

\begin{tabular}{|c|c|c|c|c|c|}
\hline & 2001 & 2002 & 2003 & 2004 & $\begin{array}{l}\text { Prel. } \\
2005\end{array}$ \\
\hline GDP at factor cost & $1,269.0$ & $1,294.6$ & $1,350.4$ & $1,420.6$ & $1,496.3$ \\
\hline Agriculture & 43.3 & 43.7 & 44.8 & 46.0 & 47.4 \\
\hline Crops & 12.6 & 12.7 & 12.9 & 13.3 & 13.7 \\
\hline Livestock & 10.3 & 10.5 & 10.9 & 11.2 & 11.4 \\
\hline Forestry & 0.8 & 0.8 & 0.8 & 0.8 & 0.8 \\
\hline Fisheries & 19.6 & 19.7 & 20.2 & 20.7 & 21.4 \\
\hline Mining and quarrying & 24.0 & 24.5 & 25.9 & 22.6 & 28.7 \\
\hline Manufacturing & 31.7 & 32.7 & 35.3 & 33.9 & 34.2 \\
\hline Electricity and water & 49.0 & 51.1 & 49.7 & 52.9 & 56.3 \\
\hline Construction & 174.1 & 180.2 & 192.3 & 197.1 & 235.5 \\
\hline Transportation & 138.0 & 133.5 & 153.1 & 175.4 & 172.7 \\
\hline Road transport & 70.7 & 65.7 & 74.4 & 85.3 & 86.9 \\
\hline Sea transport & 20.1 & 19.1 & 27.2 & 33.2 & 30.7 \\
\hline Air transport & 47.2 & 48.7 & 51.5 & 56.9 & 55.1 \\
\hline Communication & 141.6 & 142.2 & 144.2 & 148.6 & 160.5 \\
\hline Trade & 117.6 & 114.3 & 118.4 & 121.9 & 125.8 \\
\hline Hotels and restaurants & 146.8 & 149.9 & 165.3 & 176.4 & 174.5 \\
\hline Banks and insurance & 126.2 & 140.9 & 137.7 & 152.6 & 158.3 \\
\hline Housing & 93.3 & 96.1 & 99.0 & 102.5 & 106.1 \\
\hline Government services & 208.7 & 220.5 & 227.0 & 237.4 & 244.5 \\
\hline Other services & 82.5 & 85.4 & 87.2 & 89.6 & 93.4 \\
\hline \multirow[t]{2}{*}{ Less: imputed banking services } & 107.8 & 120.6 & 129.5 & 136.2 & 141.6 \\
\hline & \multicolumn{5}{|c|}{ (Annual percentage change) } \\
\hline GDP at factor cost & 1.5 & 2.0 & 4.3 & 5.2 & 5.3 \\
\hline Agriculture & -0.1 & 1.0 & 2.5 & 2.6 & 3.0 \\
\hline Crops & -2.0 & 1.0 & 1.5 & 3.0 & 3.2 \\
\hline Livestock & 1.5 & 1.9 & 4.0 & 2.7 & 2.0 \\
\hline Forestry & 0.0 & 0.0 & 0.0 & 0.0 & 0.0 \\
\hline Fisheries & 0.4 & 0.5 & 2.5 & 2.5 & 3.5 \\
\hline Mining and quarrying & 2.0 & 2.0 & 5.5 & -12.5 & 26.8 \\
\hline Manufacturing & 2.5 & 3.2 & 7.9 & -4.0 & 0.9 \\
\hline Electricity and water & 1.7 & 4.1 & -2.7 & 6.4 & 6.4 \\
\hline Construction & 4.0 & 3.5 & 6.7 & 2.5 & 19.5 \\
\hline Transportation & -3.7 & -3.3 & 14.7 & 14.6 & -1.5 \\
\hline Road transport & -2.7 & -7.1 & 13.1 & 14.7 & 2.0 \\
\hline Sea transport & -3.4 & -4.8 & 42.4 & 22.0 & -7.7 \\
\hline Air transport & -5.3 & 3.0 & 5.8 & 10.5 & -3.1 \\
\hline Communication & 5.1 & 0.4 & 1.4 & 3.1 & 8.0 \\
\hline Trade & -2.0 & -2.8 & 3.6 & 2.9 & 3.2 \\
\hline Hotels and restaurants & -7.8 & 2.1 & 10.2 & 6.8 & -1.1 \\
\hline Banks and insurance & -5.9 & 11.7 & -2.3 & 10.8 & 3.8 \\
\hline Housing & 3.5 & 3.0 & 3.0 & 3.5 & 3.5 \\
\hline Government services & 3.8 & 5.7 & 2.9 & 4.6 & 3.0 \\
\hline Other services & 1.0 & 3.5 & 2.2 & 2.7 & 4.3 \\
\hline Less: imputed banking services & -15.8 & 11.9 & 7.3 & 5.2 & 4.0 \\
\hline
\end{tabular}

Sources: Antigua and Barbuda authorities; Eastern Caribbean Central Bank; and Fund staff estimates. 
Table 3. Antigua and Barbuda: Consumer Prices, 2001-05 1/

\begin{tabular}{|c|c|c|c|c|c|c|}
\hline & Weight & 2001 & 2002 & 2003 & 2004 & 2005 \\
\hline & \multicolumn{6}{|c|}{ (End-of Period Index, January $2001=100$ ) } \\
\hline All items & 100.0 & 100.8 & 103.4 & 105.2 & 108.1 & 110.9 \\
\hline Food & 21.4 & 101.8 & 102.3 & 98.9 & 105.6 & 107.1 \\
\hline Alcoholic beverages and tobacco & 0.2 & 101.9 & 102.5 & 108.1 & 104.7 & $106 . c$ \\
\hline Clothing and footwear & 11.1 & 101.8 & 107.1 & 106.0 & 106.6 & $108 . C$ \\
\hline Accommodations & 21.8 & 101.7 & 102.3 & 107.2 & 109.7 & 109.7 \\
\hline Household furnishings & 12.6 & 101.9 & 107.2 & 111.6 & 109.1 & 114.4 \\
\hline Fuel and light & 6.4 & 86.9 & 96.1 & 94.8 & 96.1 & 113.1 \\
\hline Transportation and communication & 15.4 & 101.5 & 102.1 & 107.8 & 108.5 & 109.7 \\
\hline Medical care & 2.8 & 102.2 & 102.9 & 106.1 & 109.8 & 111.3 \\
\hline Education & 2.3 & 100.6 & 101.5 & 103.9 & 104.2 & 115.7 \\
\hline Personal services & 4.3 & 101.9 & 111.4 & 113.4 & 131.9 & 131.9 \\
\hline Other & 1.8 & 102.2 & 99.2 & 102.9 & 107.2 & 108.6 \\
\hline \multicolumn{7}{|l|}{ Special indices: } \\
\hline Energy & & 89.4 & 96.8 & 96.9 & 100.9 & 126.7 \\
\hline Energy services (electricity) & & 86.8 & 96.1 & 94.7 & 96.1 & 115.8 \\
\hline Energy commodities (LPG/cooking gas) & & 101.9 & 102.5 & 102.5 & 102.5 & 102.5 \\
\hline Energy commodities (gasoline) & & 100.0 & 100.0 & 105.9 & 121.2 & 173.6 \\
\hline All items less food & & 97.8 & 99.1 & 104.4 & 105.4 & 106.5 \\
\hline All items less energy & & 99.4 & 100.0 & 103.8 & 105.8 & 105.3 \\
\hline \multirow[t]{2}{*}{ All items less food and energy } & & 98.7 & 99.1 & 105.2 & 105.9 & 104.7 \\
\hline & \multicolumn{6}{|c|}{ (Annual percentage change) } \\
\hline All items & 100.0 & $\ldots$ & 2.6 & 1.8 & 2.8 & 2.5 \\
\hline Food & 21.4 & $\ldots$ & 0.5 & -3.4 & 6.7 & 1.5 \\
\hline Alcoholic beverages and tobacco & 0.2 & $\ldots$ & 0.6 & 5.5 & -3.1 & 2.1 \\
\hline Clothing and footwear & 11.1 & $\ldots$ & 5.2 & -1.0 & 0.6 & 1.3 \\
\hline Accommodations & 21.8 & $\ldots$ & 0.6 & 4.8 & 2.4 & 0.0 \\
\hline Household furnishings & 12.6 & $\ldots$ & 5.2 & 4.1 & -2.2 & 4.8 \\
\hline Fuel and light & 6.4 & $\ldots$ & 10.5 & -1.4 & 1.4 & 17.7 \\
\hline Transportation and communication & 15.4 & $\ldots$ & 0.6 & 5.6 & 0.6 & 1.1 \\
\hline Medical care & 2.8 & $\ldots$ & 0.7 & 3.1 & 3.5 & 1.3 \\
\hline Education & 2.3 & $\ldots$ & 0.9 & 2.3 & 0.3 & 11.0 \\
\hline Personal services & 4.3 & $\ldots$ & 9.4 & 1.8 & 16.2 & 0.0 \\
\hline Other & 1.8 & $\ldots$ & -2.9 & 3.7 & 4.2 & 1.4 \\
\hline \multicolumn{7}{|l|}{ Special indices: } \\
\hline Energy & & $\ldots$ & 8.3 & 0.1 & 4.1 & 25.6 \\
\hline Energy services (electricity) & & $\ldots$ & 10.6 & -1.4 & 1.4 & 20.5 \\
\hline Energy commodities (LPG/cooking gas) & & $\ldots$ & 0.6 & 0.0 & 0.0 & 0.0 \\
\hline Energy commodities (gasoline) & & $\ldots$ & 0.0 & 5.9 & 14.4 & 43.3 \\
\hline All items less food & & $\ldots$ & 1.3 & 5.4 & 0.9 & 1.5 \\
\hline All items less energy & & $\ldots$ & 0.6 & 3.8 & 2.0 & -0.5 \\
\hline All items less food and energy & & $\ldots$ & 0.3 & 6.2 & 0.6 & -1.1 \\
\hline
\end{tabular}

Source: Antigua and Barbuda authorities.

1/ Based on preliminary estimates of an unpublished, new consumer price index, beginning January 2001, compiled by the Statistics Division, Ministry of Finance. 
Table 4. Antigua and Barbuda: Central Government Operations, 2001-05

\begin{tabular}{|c|c|c|c|c|c|}
\hline & 2001 & 2002 & 2003 & 2004 & $\begin{array}{l}\text { Prel. } \\
2005\end{array}$ \\
\hline & \multicolumn{5}{|c|}{ (In millions of Eastern Caribbean dollars) } \\
\hline Total revenue and grants & 368.5 & 421.0 & 430.3 & 477.5 & 514.9 \\
\hline $\begin{array}{l}\text { Current revenue } \\
\text { Of which }\end{array}$ & 358.3 & 396.5 & 427.1 & 472.1 & 493.4 \\
\hline Tax revenue & 319.0 & 356.8 & 382.6 & 427.0 & 454.8 \\
\hline Capital grants & 10.2 & 24.5 & 3.2 & 5.4 & 21.4 \\
\hline Total expenditure & 606.1 & 657.9 & 624.7 & 612.1 & 683.4 \\
\hline Current expenditure & 515.3 & 558.2 & 543.5 & 556.9 & 589.2 \\
\hline Wages and salaries & 225.2 & 236.8 & 250.1 & 251.1 & 252.1 \\
\hline Employment contributions 1/ & 26.6 & 29.3 & 32.5 & 31.6 & 32.8 \\
\hline Goods and services, including utilities & 134.0 & 156.1 & 100.5 & 109.9 & 109.4 \\
\hline Interest payments & 108.0 & 102.5 & 109.6 & 108.2 & 115.0 \\
\hline External & 37.7 & 37.9 & 41.0 & 39.1 & 40.0 \\
\hline Domestic & 70.3 & 64.6 & 68.6 & 69.2 & 75.0 \\
\hline Pensions and other transfers & 21.5 & 33.5 & 50.8 & 56.0 & 79.8 \\
\hline Capital expenditure and net lending & 90.8 & 99.7 & 81.2 & 55.2 & 94.2 \\
\hline Current account balance & -157.0 & -161.7 & -116.5 & -84.8 & -95.8 \\
\hline Primary balance & -129.6 & -134.4 & -84.8 & -26.4 & -53.5 \\
\hline Overall balance & -237.6 & -236.8 & -194.5 & -134.6 & -168.6 \\
\hline Identified financing & 105.1 & 131.1 & 241.4 & 142.1 & 199.7 \\
\hline External (net) & 75.6 & 62.4 & 149.1 & 1.5 & -492.8 \\
\hline Increase in arrears & 46.8 & 51.2 & 104.4 & 23.4 & -110.3 \\
\hline External borrowing & 28.4 & 10.8 & 45.1 & -21.9 & -382.5 \\
\hline Disbursement & 92.0 & 70.7 & 114.6 & 77.0 & 28.6 \\
\hline Amortization & -63.6 & -59.9 & -69.5 & -98.9 & -411.1 \\
\hline Domestic (net) & 29.4 & 68.7 & 92.3 & 99.6 & 189.2 \\
\hline Increase in arrears 2/ & 95.9 & 75.1 & 126.5 & 117.4 & 159.5 \\
\hline ECCB & -1.6 & -8.4 & 0.2 & 0.0 & 0.0 \\
\hline Banks and others & -66.3 & -1.4 & -37.6 & -39.9 & 11.9 \\
\hline Land sales and privatization proceeds & 1.4 & 3.4 & 3.0 & 22.1 & 17.8 \\
\hline Debt relief 3/ & $\ldots$ & $\ldots$ & $\ldots$ & 41.1 & 503.4 \\
\hline \multirow[t]{2}{*}{ Statistical discrepancy } & 132.5 & 105.7 & -46.9 & -7.5 & -31.2 \\
\hline & \multicolumn{5}{|c|}{ (In percent of GDP) } \\
\hline Total revenue and grants & 19.2 & 21.7 & 21.1 & 21.6 & 21.8 \\
\hline Current revenue & 18.7 & 20.5 & 21.0 & 21.4 & 20.9 \\
\hline \multicolumn{6}{|l|}{ Of which } \\
\hline Tax revenue & 16.6 & 18.4 & 18.8 & 19.3 & 19.3 \\
\hline Capital grants & 0.5 & 1.3 & 0.2 & 0.2 & 0.9 \\
\hline Total expenditure and net lending & 31.6 & 33.9 & 30.7 & 27.7 & 28.9 \\
\hline Current expenditure & 26.9 & 28.8 & 26.7 & 25.2 & 24.9 \\
\hline Wages and salaries & 11.7 & 12.2 & 12.3 & 11.4 & 10.7 \\
\hline Employment contributions & 1.4 & 1.5 & 1.6 & 1.4 & 1.4 \\
\hline Other goods and services & 7.0 & 8.1 & 4.9 & 5.0 & 4.6 \\
\hline Interest payments & 5.6 & 5.3 & 5.4 & 4.9 & 4.9 \\
\hline Pensions and other transfers & 1.1 & 1.7 & 2.5 & 2.5 & 3.4 \\
\hline Capital expenditure and net lending & 4.7 & 5.1 & 4.0 & 2.5 & 4.0 \\
\hline Current account balance & -8.2 & -8.3 & -5.7 & -3.8 & -4.1 \\
\hline Primary balance & -6.8 & -6.9 & -4.2 & -1.2 & -2.3 \\
\hline Overall balance & -12.4 & -12.2 & -9.5 & -6.1 & -7.1 \\
\hline Identified financing & 5.5 & 6.8 & 11.9 & 6.4 & 8.5 \\
\hline External (net) & 3.9 & 3.2 & 7.3 & 0.1 & -20.9 \\
\hline Increase in arrears & 2.4 & 2.6 & 5.1 & 1.1 & -4.7 \\
\hline External borrowing (net) & 1.5 & 0.6 & 2.2 & -1.0 & -16.2 \\
\hline Disbursement & 4.8 & 3.6 & 5.6 & 3.5 & 1.2 \\
\hline Amortization & -3.3 & -3.1 & -3.4 & -4.5 & -17.4 \\
\hline Domestic (net) & 1.5 & 3.5 & 4.5 & 4.5 & 8.0 \\
\hline Increase in arrears & 5.0 & 3.9 & 6.2 & 5.3 & 6.8 \\
\hline $\operatorname{ECCB}($ net) & -0.1 & -0.4 & 0.0 & 0.0 & 0.0 \\
\hline Banks and others (net) & -3.5 & -0.1 & -1.8 & -1.8 & 0.5 \\
\hline Land sales and privatization proceeds & 0.1 & 0.2 & 0.1 & 1.0 & 0.8 \\
\hline Debt relief & $\ldots$ & $\ldots$ & $\ldots$ & 1.9 & 21.3 \\
\hline Statistical discrepancy & 6.9 & 5.5 & -2.3 & -0.3 & -1.3 \\
\hline
\end{tabular}

Sources: Antigua and Barbuda authorities; and Fund staff estimates.

$1 /$ Contributions to social security, medical benefit, and education levy.

2/ Includes interest and amortization arrears, other arrears, and unpaid employment contributions.

3/ Debt relief from a commerical supplier in 2004, and from the Italian government in 2005. 
Table 5. Antigua and Barbuda: Central Government Revenue and Grants, 2001-05

\begin{tabular}{|c|c|c|c|c|c|}
\hline & 2001 & 2002 & 2003 & 2004 & $\begin{array}{l}\text { Prel. } \\
2005\end{array}$ \\
\hline & \multicolumn{5}{|c|}{ (In millions of Eastern Caribbean dollars) } \\
\hline Total revenue and grants & 368.5 & 421.0 & 430.3 & 477.5 & 514.9 \\
\hline Current revenue & 358.3 & 396.5 & 427.1 & 472.1 & 493.4 \\
\hline Tax revenue & 319.0 & 356.8 & 382.6 & 427.0 & 454.8 \\
\hline Taxes on income & 47.7 & 61.5 & 62.6 & 46.4 & 63.8 \\
\hline Corporation tax & 43.0 & 55.5 & 57.0 & 40.4 & 38.1 \\
\hline Business tax & 1.1 & 1.3 & 0.6 & 0.4 & 0.2 \\
\hline Personal income tax & 0.1 & 0.1 & 0.2 & 0.2 & 23.4 \\
\hline Taxes on property & 5.9 & 7.9 & 11.1 & 10.0 & 10.5 \\
\hline Taxes on domestic production and consumption & 68.2 & 70.4 & 75.4 & 96.5 & 100.0 \\
\hline Hotel (bed-night) tax & 4.7 & 4.2 & 5.2 & 6.4 & 6.2 \\
\hline Guest tax (6.5 percent) & 14.3 & 13.3 & 14.6 & 22.0 & 21.9 \\
\hline Hotel guest levy ( 2 percent) & 4.0 & 3.1 & 2.9 & 5.5 & 5.5 \\
\hline Restaurant sales tax & 2.6 & 2.7 & 3.3 & 3.6 & 3.5 \\
\hline Casino tax & 0.6 & 0.6 & 0.6 & 0.6 & 0.5 \\
\hline Telecommunications tax & 9.6 & 8.7 & 8.3 & 8.6 & 9.8 \\
\hline Stamp duties & 16.1 & 18.7 & 21.4 & 27.5 & 36.3 \\
\hline Other & 16.4 & 19.0 & 19.0 & 22.2 & 16.3 \\
\hline Taxes on international transactions & 197.1 & 217.1 & 233.6 & 274.1 & 280.5 \\
\hline Consumption duties & 76.8 & 81.0 & 85.7 & 100.0 & 103.3 \\
\hline Import duty & 55.9 & 54.6 & 57.0 & 60.5 & 71.2 \\
\hline Customs service charge & 38.9 & 48.8 & 52.8 & 58.8 & 65.7 \\
\hline Travel tax & 4.0 & 4.1 & 3.6 & 4.8 & 4.5 \\
\hline Embarkation tax & 9.1 & 9.7 & 11.0 & 12.0 & 11.6 \\
\hline Foreign currency levy & 6.5 & 6.6 & 8.8 & 10.5 & 1.4 \\
\hline Cruise passenger tax & 4.2 & 3.7 & 4.0 & 5.9 & 5.0 \\
\hline Tax on gross income of offshore banks & 0.3 & 0.4 & 0.2 & 0.0 & 0.0 \\
\hline Money tansfer levy & 0.2 & 0.6 & 0.6 & 0.6 & 0.7 \\
\hline Environmental tax & 0.0 & 7.3 & 8.2 & 10.6 & 7.7 \\
\hline Other (export duties, time share) & 1.0 & 0.4 & 0.5 & 0.3 & 0.3 \\
\hline Passenger facility charge & $\ldots$ & ( & 1.1 & 10.1 & 9.2 \\
\hline Nontax revenue & 39.3 & 39.6 & 44.4 & 45.1 & 38.6 \\
\hline ECCB profits & 3.4 & 2.4 & 1.9 & 1.9 & 0.0 \\
\hline Propety leases and rents & 4.8 & 5.9 & 3.7 & 4.9 & 4.3 \\
\hline Post office revenue & 3.8 & 4.4 & 4.0 & 3.6 & 4.0 \\
\hline Other $1 /$ & 27.3 & 26.9 & 27.5 & 34.7 & 30.3 \\
\hline \multirow[t]{2}{*}{ Capital grants } & 10.2 & 24.5 & 3.2 & 5.4 & 21.4 \\
\hline & \multicolumn{5}{|c|}{ (In percent of GDP) } \\
\hline Total revenue and grants & 19.2 & 21.7 & 21.1 & 21.6 & 21.8 \\
\hline Current revenue & 18.7 & 20.5 & 21.0 & 21.4 & 20.9 \\
\hline Tax revenue & 16.6 & 18.4 & 18.8 & 19.3 & 19.3 \\
\hline Taxes on income and property & 2.8 & 3.6 & 3.6 & 2.6 & 3.1 \\
\hline Taxes on domestic production and consumption & 3.6 & 3.6 & 3.7 & 4.4 & 4.2 \\
\hline Taxes on international transactions & 10.3 & 11.2 & 11.5 & 12.4 & 11.9 \\
\hline Nontax revenue & 2.1 & 2.0 & 2.2 & 2.0 & 1.6 \\
\hline Capital grants & 0.5 & 1.3 & 0.2 & 0.2 & 0.9 \\
\hline
\end{tabular}

Sources: Antigua and Barbuda authorities; and Fund staff estimates.

$1 /$ Including taxes on services and profit-sharing from the West Indies Oil Company and surplus transfer from public enterprises. 
Table 6. Antigua and Barbuda: Central Government Expenditure, 2001-05

\begin{tabular}{|c|c|c|c|c|c|}
\hline & 2001 & 2002 & 2003 & 2004 & $\begin{aligned} \text { Prel. } \\
2005 \\
\end{aligned}$ \\
\hline & \multicolumn{5}{|c|}{ (In millions of Eastern Caribbean dollars) } \\
\hline Total expenditure and net lending & 606.1 & 657.9 & 624.7 & 612.1 & 683.4 \\
\hline $\begin{array}{l}\text { Current expenditure } \\
\text { Wages and salaries }\end{array}$ & $\begin{array}{l}515.3 \\
225.2\end{array}$ & $\begin{array}{l}558.2 \\
236.8\end{array}$ & $\begin{array}{l}543.5 \\
250.1\end{array}$ & $\begin{array}{l}556.9 \\
251.1\end{array}$ & $\begin{array}{l}589.2 \\
252.1\end{array}$ \\
\hline $\begin{array}{l}\text { Established civil servants } \\
\text { Of which }\end{array}$ & \multicolumn{4}{|c|}{ Of which } & 114.5 \\
\hline Payments in arrears & 0.0 & 0.0 & 3.1 & 5.2 & 3.6 \\
\hline \multicolumn{6}{|l|}{ Of which } \\
\hline Payments in arrears & 0.0 & 0.0 & 12.8 & 18.2 & 16.2 \\
\hline Traveling allowances $1 /$ & 5.6 & 6.0 & 5.9 & 5.7 & 26.5 \\
\hline $\begin{array}{l}\text { Other personnel costs } 1 / \\
\text { Of which }\end{array}$ & 7.1 & 14.1 & 11.1 & 14.5 & 1.5 \\
\hline Payments in arrears & 2.0 & 3.0 & 4.0 & 5.0 & 6.0 \\
\hline \multicolumn{6}{|l|}{ Of which } \\
\hline Payments in arrears & 12.3 & 13.9 & 14.1 & 13.5 & 14.0 \\
\hline $\begin{array}{l}\text { Medical benefit contributions } \\
\text { Of which }\end{array}$ & \multicolumn{4}{|c|}{ Of which } & 14.6 \\
\hline Payments in arrears & 14.3 & 11.9 & 14.4 & 13.8 & 14.6 \\
\hline Education levy & 4.0 & 3.4 & 4.0 & 3.9 & 4.2 \\
\hline \multicolumn{6}{|l|}{ Of which } \\
\hline Payments in arrears & 0.0 & 1.0 & 3.7 & 3.6 & 3.7 \\
\hline $\begin{array}{l}\text { Goods and services, including utilities } \\
\text { Of which }\end{array}$ & \multicolumn{4}{|c|}{ Of which } & 109.4 \\
\hline Payments in arrears & 20.8 & 34.0 & 33.5 & 48.7 & 37.4 \\
\hline Interest obligations & 108.0 & 102.5 & 109.6 & 108.2 & 115.0 \\
\hline Domestic & 70.3 & 64.6 & 68.6 & 69.2 & 75.0 \\
\hline \multicolumn{6}{|l|}{ Of which } \\
\hline Payments in arrears & 24.2 & 15.9 & 22.8 & 26.0 & 34.9 \\
\hline External & 37.7 & 37.9 & 41.0 & 39.1 & 40.0 \\
\hline \multicolumn{6}{|l|}{ Of which } \\
\hline Payments in arrears & 22.4 & 25.2 & 26.7 & 16.4 & 26.5 \\
\hline \multicolumn{5}{|l|}{ Of which } & 38.2 \\
\hline Payments in arrears & 0.0 & 0.0 & 6.1 & 3.1 & 0.0 \\
\hline Other transfers & 4.0 & 10.9 & 19.6 & 23.3 & 41.7 \\
\hline Private sector & 0.0 & 0.0 & 6.5 & 10.2 & 34.2 \\
\hline Regional and international & 0.0 & 6.1 & 6.7 & 8.4 & 7.4 \\
\hline Local government & 4.0 & 4.8 & 6.3 & 4.6 & 0.0 \\
\hline Capital expenditure and net lending & 90.8 & 99.7 & 81.2 & 55.2 & 94.2 \\
\hline \multirow[t]{2}{*}{ Change in unpaid capital vouchers } & 17.7 & 34.1 & 37.9 & 29.8 & 48.4 \\
\hline & \multicolumn{5}{|c|}{ (In percent of GDP) } \\
\hline Total expenditure and net lending & 31.6 & 33.9 & 30.7 & 27.7 & 28.9 \\
\hline Current expenditure & 26.9 & 28.8 & 26.7 & 25.2 & 24.9 \\
\hline Wages and salaries & 11.7 & 12.2 & 12.3 & 11.4 & 10.7 \\
\hline Goods and services, including utilities & 7.0 & 8.1 & 4.9 & 5.0 & 4.6 \\
\hline Interest payments & 5.6 & 5.3 & 5.4 & 4.9 & 4.9 \\
\hline Other & 2.5 & 3.2 & 4.1 & 4.0 & 4.8 \\
\hline Capital expenditure and net lending & 4.7 & 5.1 & 4.0 & 2.5 & 4.0 \\
\hline
\end{tabular}

Sources: Antigua and Barbuda authorities; and Fund staff estimates.

1/ Data for 2005 reflects reclassification of items from "other personnel costs" to "traveling allowance." 
Table 7. Antigua and Barbuda: Monetary Survey, 2001-05

\begin{tabular}{|c|c|c|c|c|c|}
\hline & 2001 & 2002 & 2003 & 2004 & $\begin{array}{r}\text { Prel. } \\
2005 \\
\end{array}$ \\
\hline & \multicolumn{5}{|c|}{ (In millions of Eastern Caribbean dollars, end of period) } \\
\hline Net foreign assets & 320.0 & 328.8 & 668.0 & 729.8 & $1,343.2$ \\
\hline Net imputed international reserves & 215.2 & 236.6 & 307.1 & 324.3 & 343.7 \\
\hline Commercial banks & 104.8 & 92.2 & 360.8 & 405.5 & 999.5 \\
\hline Net domestic assets & $1,133.6$ & $1,216.3$ & $1,203.1$ & $1,313.1$ & 840.9 \\
\hline Net credit to the public sector & 75.4 & 79.7 & 125.5 & 105.1 & 81.3 \\
\hline Claims on government (net) & 289.3 & 294.1 & 292.7 & 267.6 & 261.7 \\
\hline ECCB net credit to central government & 34.5 & 26.0 & 26.3 & 20.6 & 21.8 \\
\hline Commercial bank net credit to government & 254.8 & 268.1 & 266.4 & 247.0 & 239.9 \\
\hline Net credit to other public sector & -213.9 & -214.4 & -167.2 & -162.5 & -180.4 \\
\hline Credit to private sector & $1,348.7$ & $1,457.0$ & $1,491.6$ & $1,508.1$ & $1,665.6$ \\
\hline Other items (net) & -290.6 & -320.5 & -414.0 & -300.1 & -906.1 \\
\hline Money and quasi-money (M2) & $1,453.6$ & $1,545.1$ & $1,871.1$ & $2,042.9$ & $2,184.1$ \\
\hline Money & 307.1 & 308.0 & 369.5 & 444.5 & 525.3 \\
\hline Currency outside banks & 78.1 & 88.2 & 98.9 & 113.3 & 122.1 \\
\hline Demand deposits & 229.0 & 219.8 & 270.7 & 331.2 & 403.2 \\
\hline Quasi-money & $1,146.4$ & $1,237.1$ & $1,501.6$ & $1,598.4$ & $1,658.7$ \\
\hline Time deposits & 554.3 & 594.8 & 817.6 & 780.1 & 780.2 \\
\hline Savings deposits & 511.6 & 524.8 & 577.1 & 683.4 & 741.7 \\
\hline \multirow[t]{2}{*}{ Foreign currency deposits } & 80.6 & 117.5 & 106.8 & 134.8 & 136.8 \\
\hline & \multicolumn{5}{|c|}{ (12-month change in percent of $\mathrm{M} 2$ at the beginning period) } \\
\hline Net foreign assets & 12.6 & 0.6 & 21.9 & 3.3 & 30.0 \\
\hline Net imputed reserves & 3.1 & 1.5 & 4.6 & 0.9 & 0.9 \\
\hline Commercial banks net foreign assets & 9.4 & -0.9 & 17.4 & 2.4 & 29.1 \\
\hline Net domestic assets & -7.8 & 5.7 & -0.8 & 5.9 & -23.1 \\
\hline Credit to the public sector & -1.8 & 0.3 & 3.0 & -1.1 & -1.2 \\
\hline Credit to the central government & -1.0 & 0.3 & -0.1 & -1.3 & -0.3 \\
\hline Credit to the rest of the public sector & -0.8 & 0.0 & 3.1 & 0.3 & -0.9 \\
\hline Credit to the private sector & 2.9 & 7.5 & 2.2 & 0.9 & 7.7 \\
\hline Other items (net) & -8.9 & -2.1 & -6.1 & 6.1 & -29.7 \\
\hline & \multicolumn{5}{|c|}{ (12-month percentage change) } \\
\hline Credit to the private sector & 3.1 & 8.0 & 2.4 & 1.1 & 10.4 \\
\hline Money and quasi-money & 4.8 & 6.3 & 21.1 & 9.2 & 6.9 \\
\hline Money & 8.0 & 0.3 & 20.0 & 20.3 & 18.2 \\
\hline Quasi-money & 4.0 & 7.9 & 21.4 & 6.4 & 3.8 \\
\hline \multicolumn{6}{|l|}{ Memorandum item } \\
\hline Income velocity of M2 & 1.4 & 1.3 & 1.2 & 1.1 & 1.1 \\
\hline
\end{tabular}

Source: Eastern Caribbean Central Bank. 
Table 8. Antigua and Barbuda: Summary Operations of the Eastern Caribbean Central Bank, 2001-05

(In millions of Eastern Caribbean dollars)

\begin{tabular}{|c|c|c|c|c|c|}
\hline & 2001 & 2002 & 2003 & 2004 & $\begin{array}{l}\text { Prel. } \\
2005\end{array}$ \\
\hline Net (imputed) international reserves & 215.2 & 236.6 & 307.1 & 324.3 & 343.7 \\
\hline Net claims on commercial banks & -171.6 & -174.5 & -234.5 & -231.6 & -243.4 \\
\hline Claims & 0.5 & 0.4 & 0.3 & 0.3 & 0.0 \\
\hline Liabilities & 172.0 & 174.9 & 234.9 & 231.8 & 243.4 \\
\hline Currency held by banks & 31.5 & 30.8 & 36.2 & 41.1 & 47.7 \\
\hline Statutory reserves & 140.6 & 144.1 & 198.6 & 190.7 & 195.7 \\
\hline Fixed deposits & 0.0 & 0.0 & 0.0 & 0.0 & 0.0 \\
\hline Net claims on statutory bodies & 0.0 & 0.0 & 0.0 & 0.0 & 0.0 \\
\hline Net domestic assets & 34.5 & 26.0 & 26.3 & 20.6 & 21.8 \\
\hline Net credit to central government & 34.5 & 26.0 & 26.3 & 20.6 & 21.8 \\
\hline Interest on securities & 7.2 & 1.0 & 2.4 & 0.5 & 0.0 \\
\hline Temporary advances & 9.1 & 8.5 & 9.1 & 6.6 & 7.0 \\
\hline Treasury bills & 0.0 & 0.0 & 0.0 & 0.0 & 0.0 \\
\hline Debentures & 14.8 & 14.8 & 14.8 & 14.8 & 14.8 \\
\hline Other claims & 3.5 & 1.7 & 0.0 & 0.0 & 0.0 \\
\hline Central government deposits & -0.1 & 0.0 & 0.0 & -1.3 & 0.0 \\
\hline Currency in circulation & 78.1 & 88.2 & 98.9 & 113.3 & 122.1 \\
\hline Total currency issued & 109.6 & 119.0 & 135.1 & 154.5 & 169.8 \\
\hline Less: Currency held by banks & 31.5 & 30.8 & 36.2 & 41.1 & 47.7 \\
\hline
\end{tabular}

Source: Eastern Caribbean Central Bank. 
Table 9. Antigua and Barbuda: Consolidated Accounts of the Commercial Banks, 2001-05

(In millions of Eastern Caribbean dollars)

\begin{tabular}{|c|c|c|c|c|c|}
\hline & 2001 & 2002 & 2003 & 2004 & $\begin{array}{l}\text { Prel. } \\
2005\end{array}$ \\
\hline Net foreign assets & 104.8 & 92.2 & 360.8 & 405.5 & 999.5 \\
\hline Foreign assets & 505.8 & 656.3 & 802.6 & 896.3 & 1516.3 \\
\hline Foreign currency holdings & 5.2 & 9.7 & 9.0 & 9.8 & 12.3 \\
\hline Claims on ECCB area banks & 12.5 & 94.8 & 134.4 & 71.5 & 362.4 \\
\hline Claims on banks abroad & 352.2 & 388.3 & 362.2 & 466.3 & 657.6 \\
\hline Other & 136.0 & 163.5 & 296.9 & 348.6 & 484.0 \\
\hline Foreign liabilities & 401.0 & 564.1 & 441.8 & 490.8 & 516.9 \\
\hline Balances due to ECCB area banks & 87.5 & 134.9 & 84.5 & 117.2 & 59.0 \\
\hline Balances due to banks abroad & 114.7 & 125.6 & 139.2 & 167.3 & 254.6 \\
\hline Nonresident deposits & 195.9 & 298.9 & 215.5 & 200.2 & 199.7 \\
\hline Other & 2.9 & 4.7 & 2.5 & 6.1 & 3.5 \\
\hline Net position with ECCB & 159.8 & 169.8 & 211.4 & 244.8 & 242.2 \\
\hline Net claims on ECCB & 190.8 & 172.6 & 215.8 & 244.9 & 242.2 \\
\hline Currency holdings & 31.5 & 30.8 & 36.2 & 41.1 & 47.7 \\
\hline Current deposits & 159.3 & 131.8 & 179.6 & 202.5 & 192.2 \\
\hline Fixed deposits and call accounts & 0.0 & 10.0 & 0.0 & 1.2 & 2.3 \\
\hline Liabilities to ECCB & -31.0 & -2.8 & -4.4 & 0.0 & 0.0 \\
\hline Net domestic assets & $1,132.2$ & $1,222.8$ & $1,216.9$ & $1,303.1$ & 853.5 \\
\hline Net credit to government & 254.8 & 268.1 & 266.4 & 247.0 & 239.9 \\
\hline Treasury bills & 24.6 & 24.6 & 24.6 & 30.1 & 30.1 \\
\hline Other securities & 14.4 & 14.4 & 14.4 & 23.8 & 23.3 \\
\hline Loans and advances & 232.0 & 255.2 & 250.9 & 237.0 & 229.3 \\
\hline Deposits & -16.2 & -26.1 & -23.5 & -43.9 & -42.9 \\
\hline Net credit to National Insurance Scheme & -39.5 & -37.3 & -35.0 & -38.6 & -39.3 \\
\hline Net credit to public enterprises & -174.3 & -177.1 & -132.2 & -123.9 & -141.1 \\
\hline Loans, advances, and investments & 82.3 & 75.6 & 75.5 & 88.6 & 95.6 \\
\hline Deposits & -256.6 & -252.6 & -207.7 & -212.6 & -236.7 \\
\hline Net credit to other financial institutions & -63.5 & -82.9 & -70.1 & -14.2 & -35.5 \\
\hline Loans, advances, and investments & 10.1 & 8.9 & 47.3 & 54.8 & 60.4 \\
\hline Deposits & -73.6 & -91.8 & -117.4 & -69.0 & -95.9 \\
\hline Credit to private sector & $1,348.7$ & $1,457.0$ & $1,491.6$ & $1,508.1$ & $1,665.6$ \\
\hline Interbank float & 3.7 & -8.1 & -6.1 & -14.8 & 2.0 \\
\hline Claims & 45.5 & 53.6 & 32.6 & 15.0 & 58.7 \\
\hline Liabilities & -41.7 & -61.7 & -38.7 & -29.8 & -56.7 \\
\hline Net unclassified assets & -197.7 & -196.9 & -297.8 & -260.4 & -838.1 \\
\hline Liabilities to the private sector & 1375.5 & 1456.9 & 1772.2 & 1929.6 & 2061.9 \\
\hline Demand deposits & 229.0 & 219.8 & 270.7 & 331.2 & 403.2 \\
\hline Saving deposits & 511.6 & 524.8 & 577.1 & 683.4 & 741.7 \\
\hline Time deposits & 554.3 & 594.8 & 817.6 & 780.1 & 780.2 \\
\hline Foreign currency deposits & 80.6 & 117.5 & 106.8 & 134.8 & 136.8 \\
\hline
\end{tabular}

Source: Eastern Caribbean Central Bank. 
Table 10. Antigua and Barbuda: Distribution of Commercial Bank Loans and Advances, 2001-05 1/

\begin{tabular}{|c|c|c|c|c|c|}
\hline & 2001 & 2002 & 2003 & 2004 & $\begin{array}{l}\text { Prel. } \\
2005 \\
\end{array}$ \\
\hline & \multicolumn{5}{|c|}{ (In millions of Eastern Caribbean dollars) } \\
\hline Total & $1,699.9$ & $1,815.1$ & $1,887.8$ & $1,906.9$ & $2,075.8$ \\
\hline Public sector & 232.2 & 263.5 & 271.0 & 292.0 & 292.4 \\
\hline Government and public enterprises, n.i.e. & 200.4 & 234.6 & 220.5 & 224.6 & 245.9 \\
\hline Public utilities & 31.9 & 28.9 & 50.5 & 67.4 & 46.5 \\
\hline Businesses & 728.7 & 775.7 & 838.4 & 815.7 & 798.2 \\
\hline Agriculture and fisheries & 9.4 & 9.4 & 7.2 & 8.5 & 6.9 \\
\hline Manufacturing and mining & 47.2 & 47.6 & 56.2 & 57.9 & 49.5 \\
\hline Distributive trades & 210.1 & 208.6 & 196.1 & 187.4 & 190.6 \\
\hline Tourism and entertainment & 159.7 & 170.7 & 173.5 & 157.4 & 159.6 \\
\hline Transport & 29.2 & 29.6 & 34.4 & 32.5 & 34.0 \\
\hline Construction and land development & 108.8 & 126.4 & 109.8 & 133.2 & 119.3 \\
\hline Professional and other services & 131.3 & 140.9 & 197.4 & 180.9 & 194.4 \\
\hline Financial institutions & 28.9 & 37.5 & 63.7 & 57.9 & 43.7 \\
\hline Households & 738.9 & 775.8 & 778.4 & 799.2 & 985.3 \\
\hline House and land purchases & 387.7 & 393.1 & 439.6 & 456.8 & 562.3 \\
\hline Durable consumer goods & 108.3 & 79.9 & 100.8 & 95.4 & 122.4 \\
\hline \multirow[t]{2}{*}{ Other } & 243.0 & 302.8 & 238.0 & 246.9 & 300.5 \\
\hline & \multicolumn{5}{|c|}{ (In percent of total) } \\
\hline Public sector & 13.7 & 14.5 & 14.4 & 15.3 & 14.1 \\
\hline Government and public enterprises, n.i.e. & 11.8 & 12.9 & 11.7 & 11.8 & 11.8 \\
\hline Public utilities & 1.9 & 1.6 & 2.7 & 3.5 & 2.2 \\
\hline Businesses & 42.9 & 42.7 & 44.4 & 42.8 & 38.5 \\
\hline Agriculture and fisheries & 0.6 & 0.5 & 0.4 & 0.4 & 0.3 \\
\hline Manufacturing and mining & 2.8 & 2.6 & 3.0 & 3.0 & 2.4 \\
\hline Distributive trades & 12.4 & 11.5 & 10.4 & 9.8 & 9.2 \\
\hline Tourism and entertainment & 9.4 & 9.4 & 9.2 & 8.3 & 7.7 \\
\hline Transport & 1.7 & 1.6 & 1.8 & 1.7 & 1.6 \\
\hline Construction and land development & 6.4 & 7.0 & 5.8 & 7.0 & 5.7 \\
\hline Professional and other services & 7.7 & 7.8 & 10.5 & 9.5 & 9.4 \\
\hline Financial institutions & 1.7 & 2.1 & 3.4 & 3.0 & 2.1 \\
\hline Households & 43.5 & 42.7 & 41.2 & 41.9 & 47.5 \\
\hline House and land purchases & 22.8 & 21.7 & 23.3 & 24.0 & 27.1 \\
\hline Durable consumer goods & 6.4 & 4.4 & 5.3 & 5.0 & 5.9 \\
\hline Other & 14.3 & 16.7 & 12.6 & 12.9 & 14.5 \\
\hline
\end{tabular}

Source: Eastern Caribbean Central Bank.

$1 /$ Includes credits to nonresidents. 
Table 11. Antigua and Barbuda: Selected Interest Rates, 2001-05

(In percent per annum)

\begin{tabular}{|c|c|c|c|c|c|}
\hline & 2001 & 2002 & 2003 & 2004 & 2005 \\
\hline ECCB discount rate & 7.00 & 7.00 & 6.50 & 6.50 & 6.50 \\
\hline Money market rate $1 /$ & 5.64 & 6.32 & 6.07 & 4.67 & 4.01 \\
\hline Treasury bill rate ( 3 month) & 7.00 & 7.00 & 7.00 & 7.00 & 7.00 \\
\hline \multicolumn{6}{|l|}{ Commercial bank deposit rates } \\
\hline Savings & $4.00-8.00$ & $3.00-8.00$ & $3.00-8.00$ & $3.00-5.00$ & $3.00-4.25$ \\
\hline \multicolumn{6}{|l|}{ Time } \\
\hline Up to 3 months & $3.50-6.00$ & $3.20-5.75$ & $1.00-5.00$ & $1.00-4.00$ & $1.00-5.00$ \\
\hline $3-6$ months & $2.50-6.25$ & $2.50-6.00$ & $1.00-5.00$ & $1.00-5.00$ & $1.00-5.00$ \\
\hline $6-12$ months & $2.50-9.25$ & $2.50-9.25$ & $1.00-9.25$ & $1.00-7.00$ & $1.00-5.50$ \\
\hline Average deposit rate & 4.49 & 4.36 & 4.86 & 4.12 & 3.86 \\
\hline \multicolumn{6}{|l|}{ Commercial bank lending rates } \\
\hline Prime rate & $10.00-11.50$ & $10.50-11.00$ & $10.00-11.00$ & $10.00-11.50$ & $10.00-11.50$ \\
\hline Effective lending rate & $10.00-22.00$ & $10.50-22.00$ & $10.00-23.61$ & $10.20-23.61$ & $10.21-23.61$ \\
\hline Average lending rate & 11.62 & 11.39 & 12.82 & 11.96 & 11.39 \\
\hline \multicolumn{6}{|l|}{ Memorandum item: } \\
\hline $\begin{array}{l}\text { Libor on U.S. dollar six-mont } \\
\text { deposits (average) }\end{array}$ & 3.73 & 1.87 & 1.23 & 1.79 & 3.76 \\
\hline
\end{tabular}

Sources: Antigua and Barbuda authorities; Eastern Caribbean Central Bank; and Fund staff estimates.

1/ Break in the data series in 2001. 
Table 12. Antigua and Barbuda: Balance of Payments, 2001-05

\begin{tabular}{|c|c|c|c|c|c|}
\hline & 2001 & 2002 & 2003 & 2004 & $\begin{array}{l}\text { Prel. } \\
2005 \\
\end{array}$ \\
\hline & \multicolumn{5}{|c|}{$\begin{array}{l}\text { I. Balance of Payments } \\
\text { (In millions of U.S. dollars) }\end{array}$} \\
\hline Current account & -56.9 & -78.0 & -101.4 & -77.7 & -127.2 \\
\hline Trade balance & -272.8 & -269.3 & -307.6 & -317.5 & -343.7 \\
\hline Exports (f.o.b.) 1/ & 44.7 & 33.6 & 44.7 & 57.1 & 71.9 \\
\hline Imports (f.o.b.) $1 /$ & 317.5 & 303.0 & 352.4 & 374.6 & 415.5 \\
\hline Services balance & 231.6 & 227.2 & 231.3 & 270.3 & 259.4 \\
\hline \multicolumn{6}{|l|}{ Of which } \\
\hline Gross tourist receipts & 272.1 & 273.8 & 299.8 & 337.7 & 336.7 \\
\hline Income (net) & -24.8 & -41.4 & -38.6 & -39.0 & -50.9 \\
\hline \multicolumn{6}{|l|}{ Of which } \\
\hline Interest obligations & 14.0 & 14.0 & 15.2 & 14.5 & 14.8 \\
\hline Current transfers & 9.1 & 5.6 & 13.5 & 8.4 & 7.9 \\
\hline Capital and financial account & 57.1 & 92.0 & 102.1 & 63.7 & -44.9 \\
\hline Official & 14.3 & 13.1 & 17.9 & -6.1 & -133.7 \\
\hline Capital grants & 3.8 & 9.1 & 1.2 & 2.0 & 7.9 \\
\hline Borrowing & 10.5 & 4.0 & 16.7 & -8.1 & -141.7 \\
\hline Official disbursements & 34.1 & 26.2 & 42.4 & 28.5 & 10.6 \\
\hline Official amortization & 23.6 & 22.2 & 25.7 & 36.6 & 152.3 \\
\hline Non official & 42.8 & 78.9 & 84.2 & 69.8 & 88.8 \\
\hline Direct investment (net) & 98.5 & 65.9 & 166.3 & 73.6 & 123.7 \\
\hline Commercial banks & -48.5 & 4.7 & -99.5 & -16.5 & -220.0 \\
\hline Other capital & -7.1 & 8.4 & 17.4 & 12.8 & 185.1 \\
\hline Errors and omissions & -6.7 & -25.6 & -13.0 & -3.5 & 33.7 \\
\hline Overall balance & -6.4 & -11.5 & -12.3 & -17.5 & -138.4 \\
\hline Financing & 6.4 & 11.5 & 12.3 & 17.5 & 138.4 \\
\hline Change in imputed reserves (increase -) & -16.2 & -7.9 & -26.1 & -6.4 & -7.2 \\
\hline Change in government foreign assets & 0.5 & 0.5 & -0.2 & 0.0 & 0.0 \\
\hline Increase in arrears & 17.3 & 18.9 & 38.7 & 8.7 & -40.9 \\
\hline \multirow[t]{2}{*}{ Debt forgiveness } & 4.8 & 0.0 & 0.0 & 15.2 & 186.4 \\
\hline & \multicolumn{5}{|c|}{ (In percent of GDP) } \\
\hline Current account & -8.0 & -10.9 & -13.4 & -9.5 & -14.5 \\
\hline \multicolumn{6}{|l|}{ Of which } \\
\hline Trade balance & -38.4 & -37.5 & -40.8 & -38.8 & -39.3 \\
\hline Merchandise imports & 44.7 & 42.2 & 46.7 & 45.8 & 47.5 \\
\hline Gross tourist receipts & 38.3 & 38.1 & 39.8 & 41.3 & 38.5 \\
\hline $\begin{array}{l}\text { Capital and financial account } \\
\text { Of which }\end{array}$ & 8.0 & 12.8 & 13.5 & 7.8 & -5.1 \\
\hline Official disbursements (net) & 1.5 & 0.6 & 2.2 & -1.0 & -16.2 \\
\hline Direct investment (net) & 13.9 & 9.2 & 22.1 & 9.0 & 14.1 \\
\hline Errors and omissions & -0.9 & -3.6 & -1.7 & -0.4 & 3.9 \\
\hline Overall balance & -0.9 & -1.6 & -1.6 & -2.1 & -15.8 \\
\hline \multicolumn{6}{|l|}{ Memorandum items: } \\
\hline Accumulation of arrears & 2.4 & 2.6 & 5.1 & 1.1 & -4.7 \\
\hline External government debt (end of year) & 66.6 & 73.2 & 78.0 & 70.8 & 40.1 \\
\hline \multirow[t]{2}{*}{ External arrears (end of year) } & 14.5 & 19.0 & 23.2 & 22.5 & 16.4 \\
\hline & \multicolumn{5}{|c|}{$\begin{array}{l}\text { II. Tourism Indicators } \\
\text { (Annual percentage change) }\end{array}$} \\
\hline Tourist arrivals & -5.3 & -14.4 & 17.9 & 25.0 & -7.1 \\
\hline \multicolumn{6}{|l|}{ Of which } \\
\hline Air stayover arrivals & -6.6 & 2.5 & 13.1 & 9.7 & -0.2 \\
\hline Cruise ship arrivals & -4.8 & -23.6 & 22.9 & 36.2 & -10.7 \\
\hline
\end{tabular}

Sources: Antigua and Barbuda authorities; Eastern Caribbean Central Bank; and Fund staff estimates.

1/ Based on ECCB estimates. 
Table 13. Antigua and Barbuda: Selected Tourism Statistics, 2001-05

\begin{tabular}{|c|c|c|c|c|c|}
\hline & 2001 & 2002 & 2003 & 2004 & $\begin{array}{l}\text { Prel. } \\
2005 \\
\end{array}$ \\
\hline & \multicolumn{5}{|c|}{ (Number of visitors) } \\
\hline Total visitors & 641,435 & 548,833 & 646,963 & 808,497 & 751,383 \\
\hline Cruise-ship & 408,812 & 312,241 & 383,834 & 522,753 & 466,851 \\
\hline Yacht & 17,836 & 18,193 & 17,362 & 17,778 & 17,422 \\
\hline Stayovers (staying in hotels) & 171,830 & 174,719 & 187,434 & 204,364 & 203,711 \\
\hline \multirow[t]{2}{*}{ Private homes and other $1 /$} & 42,957 & 43,680 & 58,333 & 63,603 & 63,399 \\
\hline & \multicolumn{5}{|c|}{ (Percentage share of visitors) } \\
\hline Total visitors & 100.0 & 100.0 & 100.0 & 100.0 & 100.0 \\
\hline Cruise ship & 63.7 & 56.9 & 59.3 & 64.7 & 62.1 \\
\hline Yacht & 2.8 & 3.3 & 2.7 & 2.2 & 2.3 \\
\hline Stayovers (staying in hotels) & 26.8 & 31.8 & 29.0 & 25.3 & 27.1 \\
\hline \multirow[t]{2}{*}{ Private homes and other $1 /$} & 6.7 & 8.0 & 9.0 & 7.9 & 8.4 \\
\hline & \multicolumn{5}{|c|}{ (Annual percentage change) } \\
\hline Total visitors & -5.3 & -14.4 & 17.9 & 25.0 & -7.1 \\
\hline Cruise ship & -4.8 & -23.6 & 22.9 & 36.2 & -10.7 \\
\hline Yacht & 0.0 & 2.0 & -4.6 & 2.4 & -2.0 \\
\hline Stayovers (staying in hotels) & -6.6 & 1.7 & 7.3 & 9.0 & -0.3 \\
\hline \multirow[t]{2}{*}{ Private homes and other $1 /$} & -6.6 & 1.7 & 33.5 & 9.0 & -0.3 \\
\hline & \multicolumn{5}{|c|}{ (In millions of U.S. dollars) } \\
\hline Total expenditure 2/ & 272.1 & 273.8 & 299.8 & 337.7 & 336.7 \\
\hline Cruise ship & 11.4 & 8.7 & 10.7 & 15.0 & 13.4 \\
\hline Yacht & 8.2 & 8.4 & 8.0 & 8.4 & 8.3 \\
\hline Stayovers (staying in hotels) & 227.6 & 231.5 & 247.6 & 276.9 & 277.4 \\
\hline \multirow[t]{2}{*}{ Private homes and other $1 /$} & 24.8 & 25.2 & 33.5 & 37.5 & 37.6 \\
\hline & \multicolumn{5}{|c|}{ (Annual percentage change) } \\
\hline Total expenditure & -6.4 & 0.6 & 9.5 & 12.6 & -0.3 \\
\hline Cruise ship & -4.8 & -23.6 & 22.6 & 39.7 & -10.2 \\
\hline Yacht & 0.0 & 2.0 & -4.8 & 5.0 & -1.5 \\
\hline Stayovers (staying in hotels) & -6.6 & 1.7 & 7.0 & 11.8 & 0.2 \\
\hline Private homes and other $1 /$ & -6.6 & 1.7 & 33.2 & 11.8 & 0.2 \\
\hline
\end{tabular}

Sources: Antigua and Barbuda authorities; Eastern Caribbean Central Bank; and Caribbean Tourism Organization. $1 /$ Arrivals by air and sea not staying in hotels.

2/ Estimated using average expenditure from the 1995 visitor survey carried out by the Caribbean Tourism Organization. 
Table 14. Antigua and Barbuda: Air Stayover Arrivals, 2001-06

\begin{tabular}{|c|c|c|c|c|c|c|}
\hline & \multirow[b]{2}{*}{2001} & \multirow[b]{2}{*}{2002} & \multirow[b]{2}{*}{2003} & \multirow[b]{2}{*}{2004} & \multicolumn{2}{|c|}{$\begin{array}{c}\text { Prel. } \\
\end{array}$} \\
\hline & & & & & 2005 & 2006 \\
\hline & \multicolumn{6}{|c|}{ (In thousands of arrivals) } \\
\hline Total & 193.2 & 198.1 & 224.0 & 245.8 & 245.4 & $\ldots$ \\
\hline $\begin{array}{l}\text { Quarter I } \\
\text { January } \\
\text { February } \\
\text { March }\end{array}$ & $\begin{array}{l}\mathbf{5 8 . 6} \\
19.2 \\
18.8 \\
20.6\end{array}$ & $\begin{array}{l}\mathbf{5 6 . 9} \\
17.7 \\
18.2 \\
21.1\end{array}$ & $\begin{array}{l}\mathbf{5 8 . 1} \\
19.6 \\
19.0 \\
19.5\end{array}$ & $\begin{array}{l}69.1 \\
22.2 \\
23.3 \\
23.6\end{array}$ & $\begin{array}{l}75.6 \\
24.9 \\
23.7 \\
27.0\end{array}$ & $\begin{array}{l}74.2 \\
22.9 \\
24.8 \\
26.4\end{array}$ \\
\hline $\begin{array}{l}\text { Quarter II } \\
\text { April } \\
\text { May } \\
\text { June }\end{array}$ & $\begin{array}{l}\mathbf{4 9 . 8} \\
21.2 \\
14.2 \\
14.4\end{array}$ & $\begin{array}{l}\mathbf{4 7 . 0} \\
18.0 \\
15.7 \\
13.4\end{array}$ & $\begin{array}{l}56.6 \\
20.5 \\
19.4 \\
16.7\end{array}$ & $\begin{array}{l}63.5 \\
28.0 \\
18.6 \\
17.0\end{array}$ & $\begin{array}{l}\mathbf{5 9 . 1} \\
24.6 \\
18.2 \\
16.3\end{array}$ & $\begin{array}{l}63.0 \\
25.5 \\
19.7 \\
17.8\end{array}$ \\
\hline $\begin{array}{l}\text { Quarter III } \\
\text { July } \\
\text { August } \\
\text { September }\end{array}$ & $\begin{array}{r}\mathbf{4 0 . 9} \\
15.2 \\
16.0 \\
9.7\end{array}$ & $\begin{array}{l}\mathbf{4 3 . 8} \\
17.0 \\
15.7 \\
11.1\end{array}$ & $\begin{array}{l}\mathbf{5 1 . 8} \\
20.1 \\
19.9 \\
11.7\end{array}$ & $\begin{array}{l}\mathbf{5 4 . 1} \\
23.0 \\
18.1 \\
13.0\end{array}$ & $\begin{array}{l}\mathbf{5 1 . 5} \\
21.4 \\
18.0 \\
12.0\end{array}$ & $\begin{array}{l}\ldots \\
\ldots \\
\ldots \\
\ldots\end{array}$ \\
\hline $\begin{array}{l}\text { Quarter IV } \\
\text { October } \\
\text { November } \\
\text { December }\end{array}$ & $\begin{array}{l}\mathbf{4 3 . 9} \\
12.4 \\
15.1 \\
16.4\end{array}$ & $\begin{array}{l}\mathbf{5 0 . 4} \\
14.0 \\
17.8 \\
18.6\end{array}$ & $\begin{array}{l}\mathbf{5 7 . 6} \\
16.0 \\
18.4 \\
23.3\end{array}$ & $\begin{array}{l}\mathbf{5 9 . 0} \\
16.6 \\
18.6 \\
23.9\end{array}$ & $\begin{array}{l}\mathbf{5 9 . 2} \\
17.9 \\
18.4 \\
22.9\end{array}$ & $\begin{array}{l}\ldots \\
\ldots \\
\ldots \\
\ldots\end{array}$ \\
\hline December & \multicolumn{6}{|c|}{ (In percent of annual arrivals) } \\
\hline Total & 100.0 & 100.0 & 100.0 & 100.0 & 100.0 & $\ldots$ \\
\hline $\begin{array}{l}\text { Quarter I } \\
\text { January } \\
\text { February } \\
\text { March }\end{array}$ & $\begin{array}{r}30.3 \\
9.9 \\
9.7 \\
10.7\end{array}$ & $\begin{array}{r}28.7 \\
8.9 \\
9.2 \\
10.6\end{array}$ & $\begin{array}{r}25.9 \\
8.8 \\
8.5 \\
8.7\end{array}$ & $\begin{array}{r}28.1 \\
9.0 \\
9.5 \\
9.6\end{array}$ & $\begin{array}{r}30.8 \\
10.2 \\
9.7 \\
11.0\end{array}$ & $\begin{array}{l}\ldots \\
\ldots \\
\ldots \\
\ldots\end{array}$ \\
\hline $\begin{array}{l}\text { Quarter II } \\
\text { April } \\
\text { May } \\
\text { June }\end{array}$ & $\begin{array}{r}25.8 \\
11.0 \\
7.4 \\
7.5\end{array}$ & $\begin{array}{r}23.8 \\
9.1 \\
7.9 \\
6.8\end{array}$ & $\begin{array}{r}25.3 \\
9.1 \\
8.6 \\
7.5\end{array}$ & $\begin{array}{r}25.8 \\
11.4 \\
7.5 \\
6.9\end{array}$ & $\begin{array}{r}24.1 \\
10.0 \\
7.4 \\
6.6\end{array}$ & $\begin{array}{l}\ldots \\
\cdots \\
\cdots \\
\ldots\end{array}$ \\
\hline $\begin{array}{l}\text { Quarter III } \\
\text { July } \\
\text { August } \\
\text { September }\end{array}$ & $\begin{array}{r}21.2 \\
7.8 \\
8.3 \\
5.0\end{array}$ & $\begin{array}{r}22.1 \\
8.6 \\
7.9 \\
5.6\end{array}$ & $\begin{array}{r}23.1 \\
9.0 \\
8.9 \\
5.2\end{array}$ & $\begin{array}{r}22.0 \\
9.3 \\
7.4 \\
5.3\end{array}$ & $\begin{array}{r}21.0 \\
8.7 \\
7.4 \\
4.9\end{array}$ & $\begin{array}{l}\ldots \\
\ldots \\
\ldots \\
\ldots\end{array}$ \\
\hline $\begin{array}{l}\text { Quarter IV } \\
\text { October } \\
\text { November } \\
\text { December }\end{array}$ & $\begin{array}{r}22.7 \\
6.4 \\
7.8 \\
8.5\end{array}$ & $\begin{array}{r}25.4 \\
7.1 \\
9.0 \\
9.4\end{array}$ & $\begin{array}{r}25.7 \\
7.1 \\
8.2 \\
10.4\end{array}$ & $\begin{array}{r}24.0 \\
6.7 \\
7.5 \\
9.7\end{array}$ & $\begin{array}{r}\mathbf{2 4 . 1} \\
7.3 \\
7.5 \\
9.3\end{array}$ & $\begin{array}{l}\ldots \\
\ldots \\
\ldots \\
\ldots\end{array}$ \\
\hline
\end{tabular}

Sources: Antigua and Barbuda authorities; Eastern Caribbean Central Bank; and Caribbean Tourism Organization. 
Table 15. Antigua and Barbuda: Air Stayover Arrivals by Country of Origin, 2001-05

\begin{tabular}{|c|c|c|c|c|c|}
\hline & \multirow[b]{2}{*}{2001} & \multirow[b]{2}{*}{2002} & \multirow[b]{2}{*}{2003} & \multicolumn{2}{|c|}{ Prel. } \\
\hline & & & & 2004 & 2005 \\
\hline & \multicolumn{5}{|c|}{ (In thousands of arrivals) } \\
\hline Air stayover arrivals $1 /$ & 193.2 & 198.1 & 224.0 & 245.8 & 245.4 \\
\hline United States & 60.2 & 60.7 & 64.4 & 70.5 & 68.5 \\
\hline Canada & 12.8 & 10.2 & 8.6 & 9.5 & 9.9 \\
\hline Europe & 78.1 & 81.8 & 98.7 & 113.3 & 108.3 \\
\hline Caribbean & 34.3 & 37.4 & 47.6 & 48.3 & 49.0 \\
\hline Other & 7.8 & 8.0 & 4.8 & 4.1 & 9.7 \\
\hline & \multicolumn{5}{|c|}{ (In percent of air stayover arrivals) } \\
\hline United States & 31.2 & 30.6 & 28.7 & 28.7 & 27.9 \\
\hline Canada & 6.6 & 5.1 & 3.8 & 3.8 & 4.0 \\
\hline Europe & 40.4 & 41.3 & 44.0 & 46.1 & 44.1 \\
\hline Caribbean countries & 17.7 & 18.9 & 21.2 & 19.7 & 20.0 \\
\hline Other & 4.0 & 4.1 & 2.1 & 1.7 & 4.0 \\
\hline & \multicolumn{5}{|c|}{ (Average length of stay, in days) } \\
\hline Visitors staying in hotels & 7.8 & 7.8 & 7.8 & 7.8 & 7.8 \\
\hline
\end{tabular}

Sources: Antigua and Barbuda authorities; Eastern Caribbean Central Bank; and Caribbean Tourism Organization.

$1 /$ Includes stayovers in hotels, private residences, etc. 
Table 16. Antigua and Barbuda: Visitor Arrivals as a Share of the Caribbean Area, 2001-05 1/

\begin{tabular}{|c|c|c|c|c|c|}
\hline & 2001 & 2002 & 2003 & 2004 & $\begin{array}{l}\text { Prel. } \\
2005 \\
\end{array}$ \\
\hline & \multicolumn{5}{|c|}{ (In thousands, unless otherwise indicated) } \\
\hline \multicolumn{6}{|l|}{ Stayover passenger arrivals $2 /$} \\
\hline Antigua and Barbuda & 215 & 218 & 246 & 268 & 267 \\
\hline Caribbean area & 16,206 & 15,736 & 17,207 & 18,313 & 18,647 \\
\hline Antiguan share of market (in percent) & 1.3 & 1.4 & 1.4 & 1.5 & 1.4 \\
\hline \multicolumn{6}{|l|}{ Cruise ship passenger arrivals } \\
\hline Antigua and Barbuda & 409 & 312 & 384 & 523 & 467 \\
\hline Caribbean area & 13,964 & 14,347 & 17,257 & 18,792 & 18,106 \\
\hline Antiguan share of market (in percent) & 2.9 & 2.2 & 2.2 & 2.8 & 2.6 \\
\hline & \multicolumn{5}{|c|}{ (Annual percentage change) } \\
\hline \multicolumn{6}{|l|}{ Stayover passenger arrivals $2 /$} \\
\hline Antigua and Barbuda & -6.6 & 1.7 & 12.5 & 9.0 & -0.3 \\
\hline Caribbean area & -20.7 & -2.9 & 9.3 & 6.4 & 1.8 \\
\hline \multicolumn{6}{|l|}{ Cruise ship passenger arrivals } \\
\hline Antigua and Barbuda & -4.7 & -23.6 & 22.9 & 36.2 & -10.7 \\
\hline Caribbean area & 2.3 & 2.7 & 20.3 & 8.9 & -3.7 \\
\hline
\end{tabular}

Sources: Antigua and Barbuda authorities; Eastern Caribbean Central Bank; and Caribbean Tourism Organization.

1/ Comprising 32 countries and dependent territories monitored by the Caribbean Tourism Organization.

$2 /$ Including stayover arrivals by sea and air. 
Table 17. Antigua and Barbuda: Public and Publicly Guaranteed External Debt, 2001-05

\begin{tabular}{|c|c|c|c|c|c|}
\hline & 2001 & 2002 & 2003 & 2004 & $\begin{array}{l}\text { Prel. } \\
2005\end{array}$ \\
\hline & \multicolumn{5}{|c|}{ (In millions of U.S. dollars) } \\
\hline Outstanding debt (beginning of year) $1 /$ & 449.3 & 473.0 & 525.8 & 588.2 & 579.1 \\
\hline Central government & 414.7 & 440.3 & 491.6 & 557.4 & 550.3 \\
\hline Government guaranteed & 34.6 & 32.7 & 34.2 & 30.7 & 28.8 \\
\hline Disbursements & 34.1 & 26.2 & 42.4 & 28.5 & 10.6 \\
\hline Central government & 33.4 & 24.0 & 39.2 & 27.7 & 10.6 \\
\hline Government guaranteed & 0.7 & 2.1 & 3.3 & 0.8 & 0.0 \\
\hline Cash amortization & 14.5 & 12.6 & 10.9 & 41.4 & 17.5 \\
\hline Central government $2 /$ & 13.7 & 11.0 & 8.1 & 38.2 & 15.9 \\
\hline Government guaranteed & 0.9 & 1.5 & 2.9 & 3.2 & 1.6 \\
\hline Increase in interest arrears & 7.9 & 14.9 & 21.7 & 14.1 & -25.7 \\
\hline Central government & 7.7 & 14.7 & 17.8 & 13.5 & -26.1 \\
\hline Government guaranteed 3/ & 0.2 & 0.2 & 3.9 & 0.5 & 0.4 \\
\hline Stock adjustments $4 /$ & -3.7 & 24.3 & 9.1 & -10.3 & -195.5 \\
\hline Central government & -1.8 & 23.6 & 17.0 & -10.1 & -194.6 \\
\hline Government guaranteed & -2.0 & 0.7 & -7.9 & -0.2 & -0.9 \\
\hline Outstanding debt (end of year) & 473.0 & 525.8 & 588.2 & 579.1 & 351.0 \\
\hline Central government & 440.3 & 491.6 & 557.4 & 550.3 & 324.4 \\
\hline Government guaranteed & 32.7 & 34.2 & 30.7 & 28.8 & 26.6 \\
\hline Scheduled debt-service obligations 3 / & 37.5 & 36.2 & 40.9 & 51.1 & 167.1 \\
\hline Interest & 14.0 & 14.0 & 15.2 & 14.5 & 14.8 \\
\hline \multicolumn{6}{|l|}{ Of which } \\
\hline Central government & 13.7 & 13.6 & 15.2 & 13.4 & 13.9 \\
\hline Principal & 23.6 & 22.2 & 25.7 & 36.6 & 152.3 \\
\hline \multicolumn{6}{|l|}{ Of which } \\
\hline Central government & 22.7 & 20.6 & 23.5 & 34.5 & 149.7 \\
\hline Cash payments on debt obligations $2 /$ & 20.2 & 17.2 & 16.2 & 49.8 & 22.5 \\
\hline Interest & 5.7 & 4.7 & 5.3 & 8.4 & 5.0 \\
\hline \multicolumn{6}{|l|}{ Of which } \\
\hline Central government & 5.4 & 4.2 & 4.9 & 8.1 & 4.7 \\
\hline Principal & 14.5 & 12.6 & 10.9 & 41.4 & 17.5 \\
\hline \multicolumn{6}{|l|}{ Of which } \\
\hline Central government & 13.7 & 11.0 & 8.1 & 38.2 & 15.9 \\
\hline Stock of arrears (end of year) 5/ & 103.2 & 136.6 & 175.3 & 184.0 & 143.1 \\
\hline Interest arrears & 62.1 & 77.0 & 98.7 & 112.8 & 87.1 \\
\hline \multicolumn{6}{|l|}{ Of which } \\
\hline Central government & 59.4 & 74.1 & 91.9 & 105.4 & 79.3 \\
\hline Principal arrears & 41.1 & 59.6 & 76.6 & 71.2 & 56.0 \\
\hline \multicolumn{6}{|l|}{ Of which } \\
\hline \multirow[t]{2}{*}{ Central government } & 32.8 & 50.7 & 65.2 & 60.8 & 45.6 \\
\hline & \multicolumn{5}{|c|}{ (In percent of total outstanding debt) } \\
\hline \multicolumn{6}{|l|}{ Debt by creditor } \\
\hline Multilateral creditors & 1.9 & 1.8 & 2.3 & 2.5 & 3.6 \\
\hline Bilateral creditors & 63.9 & 63.0 & 49.3 & 50.2 & 29.8 \\
\hline Commercial creditors & 34.3 & 35.2 & 48.5 & 47.3 & 66.6 \\
\hline \multicolumn{6}{|l|}{ Debt indicators } \\
\hline \multirow{2}{*}{\multicolumn{6}{|c|}{$\begin{array}{l}\text { Scheduled debt service/exports } \\
\text { of goods and services }\end{array}$}} \\
\hline & 8.4 & 8.5 & 8.9 & 9.7 & 30.8 \\
\hline \multicolumn{6}{|l|}{ Scheduled debt service/central } \\
\hline government revenue and grants & 27.5 & 23.2 & 25.7 & 28.9 & 87.6 \\
\hline Cash payments/scheduled obligations & 53.9 & 47.7 & 39.7 & 97.4 & 13.5 \\
\hline Debt outstanding/GDP & 66.6 & 73.2 & 78.0 & 70.8 & 40.1 \\
\hline \multicolumn{6}{|l|}{ Of which } \\
\hline Stock of arrears/GDP & 14.5 & 19.0 & 23.2 & 22.5 & 16.4 \\
\hline Average implied interest rate & 3.0 & 2.7 & 2.6 & 2.5 & 4.2 \\
\hline
\end{tabular}

Sources: Antigua and Barbuda authorities; Eastern Caribbean Central Bank; and Fund staff estimates.

$1 /$ Including arrears. Debt at beginning of year equals debt outstanding at end of preceding year.

2/ Includes cash payments to the Italian government in 2004-05 and to a commercial supplier in 2004.

3 / For government guaranteed debt, scheduled debt service is assumed to equal cash debt service before 2004, due to data constraints.

4/ Including exchange rate valuation adjustments and other adjustments mainly due to debt relief and rescheduling.

$5 /$ Including interest arrears. 
Table 18. Antigua and Barbuda: Effective Exchange Rate Indices, 1990-2006 $(2000=100 ;$ period averages $)$

\begin{tabular}{|c|c|c|c|c|}
\hline & $\begin{array}{c}\text { Real Effective } \\
\text { Exchange Rate } \\
\text { Index }\end{array}$ & $\begin{array}{c}\text { Nominal Effective } \\
\text { Exchange Rate } \\
\text { Index }\end{array}$ & $\begin{array}{c}\text { Consumer } \\
\text { Price } \\
\text { Index }\end{array}$ & $\begin{array}{l}\text { Relative } \\
\text { Price } \\
\text { Index }\end{array}$ \\
\hline 1990 & 104.6 & 91.4 & 85.5 & 114.5 \\
\hline 1991 & 106.9 & 92.5 & 90.4 & 115.6 \\
\hline 1992 & 105.5 & 92.5 & 93.1 & 114.0 \\
\hline 1993 & 111.7 & 98.3 & 95.7 & 113.7 \\
\hline 1994 & 102.7 & 98.3 & 90.6 & 104.5 \\
\hline 1995 & 99.1 & 95.3 & 92.7 & 104.0 \\
\hline 1996 & 100.9 & 96.9 & 95.4 & 104.1 \\
\hline 1997 & 100.9 & 98.9 & 95.9 & 102.1 \\
\hline 1998 & 104.2 & 100.8 & 98.8 & 103.3 \\
\hline 1999 & 102.0 & 99.4 & 100.0 & 102.7 \\
\hline 2000 & 100.0 & 100.0 & 100.0 & 100.0 \\
\hline 2001 & 103.3 & 102.5 & 103.3 & 100.9 \\
\hline 2002 & 104.7 & 102.2 & 106.5 & 102.5 \\
\hline 2003 & 102.4 & 98.9 & 109.8 & 103.5 \\
\hline 2004 & 100.2 & 96.2 & 113.0 & 104.1 \\
\hline 2005 & 99.9 & 96.0 & 116.3 & 104.0 \\
\hline 2001 I & 102.1 & 101.8 & 102.2 & 100.3 \\
\hline 2001 II & 103.4 & 102.9 & 102.9 & 100.5 \\
\hline $2001 \mathrm{III}$ & 103.3 & 102.5 & 103.5 & 100.8 \\
\hline 2001 IV & 104.6 & 102.7 & 104.5 & 101.8 \\
\hline 2002 I & 106.3 & 104.0 & 105.4 & 102.3 \\
\hline 2002 II & 105.1 & 102.7 & 106.1 & 102.4 \\
\hline 2002 III & 103.3 & 100.9 & 106.7 & 102.4 \\
\hline 2002 IV & 104.1 & 101.2 & 107.8 & 102.8 \\
\hline 2003 I & 102.8 & 100.0 & 108.6 & 102.8 \\
\hline 2003 II & 102.7 & 99.3 & 109.4 & 103.4 \\
\hline 2003 III & 102.8 & 99.2 & 110.2 & 103.7 \\
\hline 2003 IV & 101.2 & 97.3 & 111.0 & 104.1 \\
\hline I & 100.1 & 96.1 & 111.8 & 104.1 \\
\hline 2004 II & 100.7 & 96.9 & 112.6 & 104.0 \\
\hline 2004 III & 100.7 & 96.6 & 113.4 & 104.2 \\
\hline 2004 IV & 99.2 & 95.3 & 114.2 & 104.0 \\
\hline | & 98.9 & 94.8 & 115.1 & 104.2 \\
\hline 2005 II & 99.6 & 95.6 & 115.9 & 104.2 \\
\hline $2005 \mathrm{III}$ & 100.1 & 96.4 & 116.7 & 103.9 \\
\hline 2005 IV & 100.9 & 97.2 & 117.6 & 103.8 \\
\hline 2006 I & 100.8 & 96.9 & 118.4 & 104.0 \\
\hline 2006 II & 99.3 & 95.9 & 119.3 & 103.6 \\
\hline
\end{tabular}

Source: IMF, Information Notice System. 